\title{
Reparación de sección de arteria axilar por trauma vascular secundario a herida por arma blanca: a propósito de un caso
}

\author{
Consuelo Santibáñez P. ${ }^{1,2}$, Sophia Cottin B..$^{1,2}$, \\ Karen Schonffeldt G. ${ }^{1,2}$ y Juan Francisco Allamand T. ${ }^{2}$
}

'Facultad de Medicina Clínica

Alemana de SantiagoUniversidad del Desarrollo. 2Servicio de Cirugía, Hospita Padre Hurtado.

Recibido el 13 de julio de 2017 y aceptado para publicación el 3 de agosto de 2017

Correspondencia a: Dr. Juan Francisco Allamand juanfcoallamand@gmail.com

\section{Repair of axillary artery section due to vascular trauma secondary to stab wounds: a case report}

Objective: To present a clinical case and discuss the management of axillary artery trauma and to review current literature. Clinical case: Male patient with axillary artery injury, who is brought in hypovolemic shock to the Emergency Department. Surgical findings demonstrated an incomplete section of the second portion of the axillary artery which was repaired with primary end-to-end anastomosis. Postoperatively, the patient evolves without pain and is discharged. Discussion: The management of vascular trauma should be methodical and multidisciplinary. Hard signs of vascular trauma mandate immediate surgical exploration. Patients with soft signs may need further evaluation with images. In an incomplete section of the axillary artery the end-to-end anastomosis has shown good results. Conclusions: Axillary artery lesions in the context of trauma are infrequent. Its management requires a high index of suspicion, a multidisciplinary approach and adequate surgical access.

Key words: vascular trauma, axillary artery.

\section{Resumen}

Objetivo: Presentar un caso clínico y discutir el manejo del trauma de arteria axilar y revisar la literatura actual. Caso Clínico: se presenta el caso de un paciente con lesión de arteria axilar, que es traído en shock hipovolémico al Servicio de Emergencias de nuestro hospital. En pabellón, se aprecia sección incompleta de la segunda porción de la arteria axilar. Se controla y repara con anastomosis primaria término-terminal. En su posoperatorio evoluciona de forma satisfactoria. Discusión: El manejo del trauma vascular debe ser metódico y multidisciplinario. Los signos duros de trauma vascular son indicación de exploración quirúrgica inmediata. Los pacientes con signos blandos pueden complementarse con imágenes. En una sección incompleta de arteria axilar, la anastomosis término-terminal ha mostrado buenos resultados. Conclusiones: las lesiones de arteria axilar en el contexto de traumas son infrecuentes. Su manejo necesita de un alto índice de sospecha, un enfoque multidisciplinario y un acceso quirúrgico adecuado al contexto.

Palabras clave: trauma vascular; arteria axilar.

\section{Introducción}

El trauma vascular puede tener graves consecuencias, tales como la amputación de una extremidad o la muerte del paciente ${ }^{1-4}$. Las lesiones vasculares de extremidades superiores representan el 30\% de todos los traumas vasculares ${ }^{4,5}$, siendo la principal etiología el trauma penetrante ${ }^{2,6}$. De estos, las lesiones de arteria axilar o subclavia son poco frecuen- tes ${ }^{1,4,6,7}$ representando menos de un $5 \%$ de todos los traumas vasculares en civiles, sin embargo, a pesar de su limitada frecuencia, estas lesiones se asocian a tasas de morbilidad y mortalidad que van del $5 \%$ a $39 \%$. Entre un $6 \%$ y un $15 \%{ }^{3,4}$ ocurren por trauma cerrado, típicamente luego de una luxación traumática de hombro ${ }^{1}$. La exposición de vasos subclavios o axilar está asociada con una morbilidad significati$\mathrm{va}^{7,8}$ y la mortalidad oscila entre el $5 \%$ y el $30 \%{ }^{6,8,9}$. 
Entre un $27 \%$ y un $44 \%{ }^{10}$ de las lesiones de arteria axilar se acompañan de lesiones del plexo braquial, principal indicador de morbilidad a largo plazo ${ }^{1,7}$. Otras lesiones concomitantes son el trauma de vena axilar en un $30 \%-43 \%{ }^{7}$ y neumotórax o hemotórax en un 18\%-29\%, además de lesiones del tracto aerodigestivo, cadena simpática y médula espinal ${ }^{9}$.

Presentamos a continuación el caso de un paciente con trauma de arteria axilar por herida por arma blanca y una revisión del tema.

\section{Descripción del caso}

Paciente de sexo masculino de 30 años de edad, consulta con herida corto-punzante en hombro izquierdo. Ingresa al box de reanimación y se monitoriza, donde se constata shock hipovolémico. Se inicia volemización y manejo general del trauma. El examen físico y la ecografía sugieren hemotórax izquierdo, por lo que se instala pleurostomía. La ecografía descarta derrame pericárdico. Al cabo de la reanimación inicial, la lesión previamente descrita evoluciona con sangrado arterial activo y hematoma expansivo, antes ausentes. Se decide cirugía de urgencia.

En pabellón, se realiza incisión infraclavicular transversa para exponer la arteria axilar distal. Dado la dificultad para lograr el control vascular, se extiende incisión hacia fosa supraclavicular, seccionando y resecando el tercio medio de la clavícula. Se evidencia así una sección incompleta de la segunda porción de la arteria axilar izquierda, por posterior al músculo pectoral menor (Figura 1).

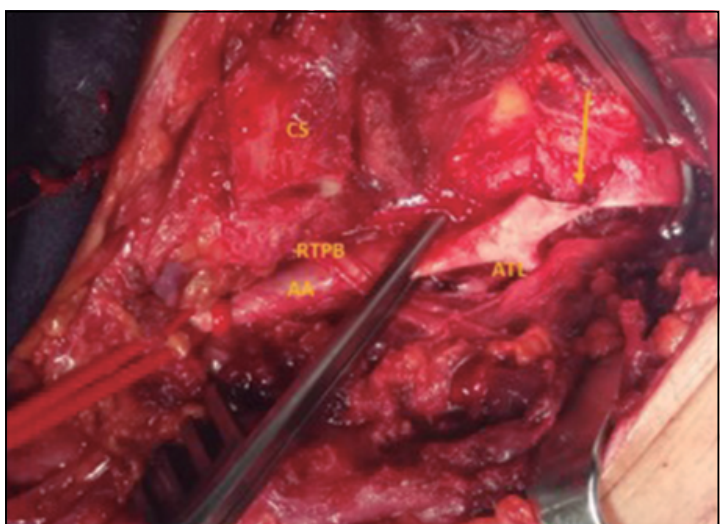

Figura 1. Trauma de arteria axilar izquierda por arma blanca con sección parcial de su segunda porción (flecha). Acceso supraclavicular con sección de clavícula y posterior control vascular proximal y distal. CS: Clavícula seccionada, AA: Arteria axilar, RTPB: Ramo terminal plexo braquial, ATL: Arteria torácica lateral.
Una vez logrado el control vascular, se realiza heparinización local, se reavivan los bordes arteriales, y se realiza una anastomosis término-terminal con sutura de polipropileno 7.0 (Figura 2). Se afrontan planos musculares, cubriendo reparación y se fija segmento de clavícula resecado a ambos extremos con sutura de polipropileno por falta de material de osteosíntesis adecuado.

En el posoperatorio el paciente evoluciona de manera favorable, con buena hemodinamia. Recupera el pulso radial de la extremidad comprometida, con movilidad y sensibilidad conservadas. Al control ambulatorio, el paciente se encuentra sin dolor, tiene pulsos presentes, sin pseudoartrosis y con buena movilidad de la extremidad.

\section{Discusión}

El trauma vascular debe ser identificado y manejado de manera eficiente, de lo contrario puede conducir a la pérdida de una extremidad o hasta la vida del paciente ${ }^{1-4}$. Para esto es primordial no retrasar el diagnóstico ${ }^{4}$. Éste se basa en el examen físico y en ocasiones de exámenes complementarios ${ }^{4}$. Los signos duros como sangrado o hematoma pulsátil, ausencia de pulso a distal y signos de isquemia aguda $^{1,3,10}$, son eficientes en confirmar el trauma vascular con un valor predictivo positivo de casi el $100 \%{ }^{2}$, pero su ausencia no lo excluye completamente $^{1,2}$. En alrededor de un tercio de las lesiones puede encontrarse pulso ya que el lumen arterial no se compromete por completo o se mantiene gracias a la circulación colateral existente ${ }^{1}$. Esto ocurre en

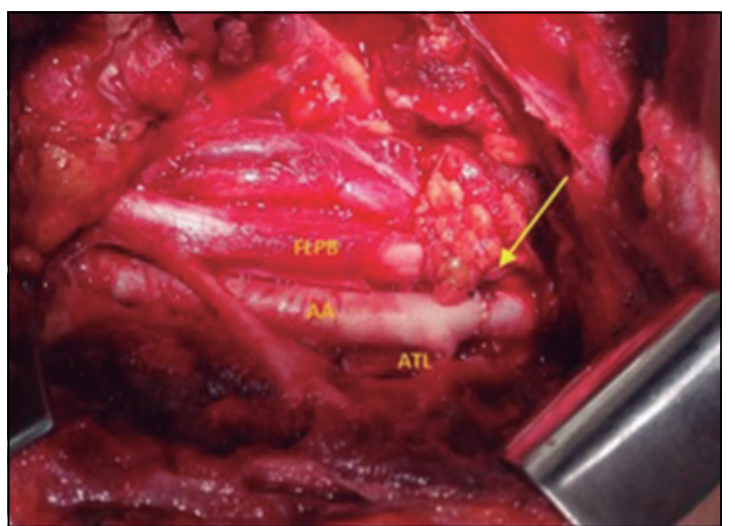

Figura 2. Reparación de arteria axilar mediante anastomosis primaria con Prolene 7.0. Se retira control vascular proximal y distal, evidenciándose ausencia de filtrado por anastomosis (flecha). FLPB: Fascículo lateral plexo braquial, AA: Arteria axilar, ATL: Arteria torácica lateral. 
lesiones de arteria axilar dado que existe una extensa red de anastomosis alrededor del hombro ${ }^{1,5,11}$, lo que en ocasiones retrasa el diagnóstico. En variados reportes de casos, la ausencia de pulso estuvo hasta en un $20 \%$ de los $\operatorname{casos}^{1}$. Cuando esto ocurre, se deben buscar signos blandos como herida en vecindad al trayecto de vasos mayores, sangrado transitorio luego del accidente, pulsos distales disminuidos con baja perfusión a distal o formación de un hematoma no pulsátil ${ }^{1,3,7,10}$. Los signos duros o de inestabilidad hemodinámica son indicación de exploración quirúrgica de urgencia ${ }^{3,5}$, mientras que los signos blandos pueden complementarse con imágenes como el ultrasonido, la tomografía o la angiografía ${ }^{1,3,10}$.

La evaluación multidisciplinaria y metódica guiada por métodos como ATLS, permiten una aproximación diagnóstica inicial, una reanimación adecuada y guiar el tratamiento ${ }^{3,10}$. Así, se identifican pacientes candidatos a cirugía de urgencia bajo los criterios ya mencionados.

Respecto a las técnicas de reparación vascular, se debe elegir entre cirugía abierta o técnica endovascular, siendo la cirugía abierta hasta hoy el Gold Standard $^{4,12}$. Se recomienda una incisión amplia que permita adecuada visualización para el control vascular proximal y distal ${ }^{8,10}$. Una vez logrado el control vascular y con visualización óptima de la lesión, se procede a la reparación. Dependiendo del tipo y extensión del daño puede preferirse una arteriorrafia, anastomosis primaria o la colocación de un injerto que puede ser de material autólogo o protésico ${ }^{1,5}$. Lesiones focales, puntiformes, serán susceptibles de reparar mediante rafia simple ${ }^{10}$, pero cuando existen secciones completas o incompletas, se debe recurrir a alternativas. Si es posible aproximar los cabos sin tensión, es preferible realizar una anastomosis primaria $^{1,2}$. En un reporte realizado por McCready et $\mathrm{al}^{1}$, se evaluaron 9 anastomosis primarias de arteria subclavia y 11 de arteria axilar, de los cuales solo 1 tuvo fallas. En otros reportes, la reparación primaria de arteria subclavia y axilar también ha mostrado excelentes resultados ${ }^{1}$.

Cuando el trauma es secundario a una herida por arma de fuego habrá pérdida de un mayor segmento arterial, por lo que se requerirá el uso de injertos $^{1,4,10,11}$. Los injertos protésicos se asocian a mayor riesgo de infección ${ }^{1,4,10,13}$ y trombosis, lo cual aumenta el riesgo de amputaciones secundarias ${ }^{13}$. Varios autores de estudios retrospectivos han preferido la utilización de injertos autólogos, a pesar de que no hay consenso absoluto al respecto ${ }^{1}$.

Los resultados de la reparación arterial a largo plazo son excelentes ${ }^{1}$. Destaca su baja tasa de fasciotomías, dado que no suele producirse una isquemia suficiente para generar un síndrome compartimental $^{5}$. Por lo mismo el riesgo de amputación es menor que en extremidades inferiores y cuando ocurre suele deberse a un uso prolongado de torniquete ${ }^{5}$. Ahora bien, a pesar de sus excelentes resultados, la reparación vascular por cirugía convencional no está exenta de riesgos ${ }^{4}$. Existen sitios anatómicos complejos, donde el abordaje quirúrgico prolonga el tiempo de isquemia o aumenta el riesgo de lesiones vasculares o nerviosas ${ }^{4,8,10,14,15}$. En traumas axilosubclavios, la aproximación por técnica abierta se enfrenta al riesgo de lesiones del plexo braquial ${ }^{1}$, nervio frénico, vago o laríngeo recurrente ${ }^{5}$, los cuales tienen alto impacto en la morbilidad ${ }^{1,8,9,14}$. Es aquí donde la terapia endovascular pudiera aportar desarrollo futuro, permitiendo accesos mínimamente invasivos y menor morbilidad en casos seleccio$\operatorname{nados}^{6,8,10,14}$. Según reportes recientes, la terapia endovascular reduciría la magnitud del sangrado, el tiempo quirúrgico, tiempo de estadía hospitalaria y riesgo de lesiones iatrogénicas ${ }^{6,8,9,14}$, pero aumentaría considerablemente los $\operatorname{costos}^{10}$. Su utilización está en alza ${ }^{3,8,10,12,14,15}$, especialmente en pacientes con múltiples lesiones, comorbilidades o malos candidatos a anestesia general ${ }^{9,11}$. En un análisis retrospectivo realizado por Branco et $\mathrm{al}^{5}$, se analizaron 23.105 pacientes con trauma vascular penetrante o cerrado entre el 2002 y 2010, observándose un aumento del uso de la terapia endovascular de un $0,3 \%$ a un $9 \%$ en ese período. Junto a esto, la mortalidad disminuyó desde un $28,2 \%$ a un $13,1 \%$. Esta alternativa se asoció a menores tasas de infección de herida quirúrgica, sepsis y amputaciones mayores ${ }^{5}$.

Si bien el uso de la terapia endovascular está en alza y ha demostrado excelentes resultados a corto plazo $^{1,9,14}$, aún no existen estudios clínicos randomizados que comparen la alternativa endovascular con la cirugía abierta, ni tampoco hay estudios que evalúen sus resultados a largo plazo ${ }^{5,12}$. Es probable que esto no ocurra pronto, especialmente en lesiones axilares y subclavias que son infrecuentes dentro de los traumas vasculares 9 . Ahora bien, resulta evidente que no todas las lesiones pueden ser candidatas a una resolución endovascular ${ }^{4,14}$. Danetz et $\mathrm{al}^{8}$ intentaron definir las principales contraindicaciones para el manejo endovascular, que incluían pérdida de segmentos arteriales mayores, secciones completas o incompletas e inestabilidad hemodinámica ${ }^{7,12}$, entre otros. Evidentemente, lesiones limitadas como laceraciones focales ${ }^{6}$, pseudoaneurismas o fístulas arteriovenosas serán las principales indicaciones ${ }^{2,4,5,8}$.

Luego de una reparación arterial existe un 35\% de riesgo de complicaciones postoperatorias, según lo documentado en un estudio de Danetz et $\mathrm{al}^{8}$, 
tales como trombosis, filtración o infección de la herida ${ }^{1}$, siendo estas últimas dos las más frecuentes reportadas en la literatura ${ }^{8}$. Aún si la reparación fue adecuada, la isquemia previa puede determinar la inviabilidad de los tejidos. En una revisión sistemática que incluyó 45 estudios, con un total de 3.187 reparaciones por trauma vascular de extremidades inferiores, se observó que algunos de los factores que aumentaban el riesgo de amputación secundaria son la isquemia mayor a $6 \mathrm{~h}$, lesiones múltiples y etiología explosiva, entre otros ${ }^{8}$. Por esto, tanto en reparaciones convencionales como en técnicas endovasculares, los pacientes deben ser observados por un tiempo prudente.

\section{Conclusión}

Las lesiones axilares en el contexto de trauma son infrecuentes y difíciles de diagnosticar, por lo que su diagnóstico necesita un alto índice de sospecha y un manejo multidisciplinario. Se deben tener en cuenta múltiples variables, lo que dificulta la posibilidad de crear un criterio de selección único para la reparación. Existen estudios retrospectivos que comparan la terapia abierta con la endovascular ${ }^{15}$, en los cuales existe el sesgo de quien decide el manejo quirúrgico es el cirujano que está en ese momento con el paciente, de acuerdo a su experiencia. Por estas razones, por ahora la cirugía abierta sigue siendo el Gold Standard de tratamiento, pero a medida que aumenten los recursos y capacitaciones de terapia endovascular, se volverá una opción cada vez más realizable.

\section{Responsabilidades éticas}

Protección de personas y animales. Los autores declaran que para esta investigación no se han realizado experimentos en seres humanos ni en animales.

Confidencialidad de los datos. Los autores declaran que han seguido los protocolos de su centro de trabajo sobre la publicación de datos de pacientes.

Derecho a la privacidad y consentimiento informado. Los autores han obtenido el consentimiento informado de los pacientes y/o sujetos referidos en el artículo. Este documento obra en poder del autor de correspondencia.

\section{Conflicto de intereses}

Los autores declaran no tener conflictos de interés.

\section{Agradecimientos}

Los autores agradecen al Servicio de Cirugía del Hospital Padre Hurtado y a la Facultad de Medicina Clínica Alemana - Universidad del Desarrollo, por su continuo apoyo a la investigación y la academia.

\section{Bibliografía}

1. McCready, R, Procter C, Hyde G. Subclavian-axillary vascular trauma. Journal of Vascular Surgery 1986;3:2431. Recuperado el 23 de abril de 2017, de http://www.jvascsurg.org/ article/0741-5214(86)90065-0/fulltext

2. Gill H, Jenkins W, Edu S, Bekker W. Civilian Penetrating Axillary Artery Injuries. World Journal of Surgery. 2011;35:962-6. Recuperado el 10 de mayo de 2017, de https://www.researchgate. net/profile/Pradeep_Navsaria/ publication/50229312_Civilian Penetrating Axillary Artery Injuries/ links/02bfe5108f9fb0a351000000/ Civilian-Penetrating-Axillary-ArteryInjuries.pdf

3. Gil F, Rojas, A, Pozzo A, Trueba C,
Pino J. Complicaciones de la luxación traumática de hombro. Ruptura parcial de la Arteria Axilar y lesión del plexo braquial. Asociación Mexicana de Medicina y Cirugía de Trauma. 2003;6:558. Recuperado el 27 de mayo de 2017, de http://www.medigraphic.com/pdfs/trauma/ tm-2003/tm032d.pdf

4. Salas C. (2011). Trauma Vascular, Visión del Cirujano Vascular. Revista Médica Clínica Las Condes. 2011;22:686-95. Recuperado el 20 de abril de 2017, de http://www.sciencedirect.com/science/ article/pii/S0716864011704813

5. Xenos ES, Freeman M, Stevens S, Cassada D, Pacanowski J, Goldman M. (2003). Covered stent for injuries of the subclavian and axillary arteries. Journal of Vascular Surgery. 2003;38:451-4. Recuperado el 10 de mayo de 2017, de
http://www.jvascsurg.org/article/S07415214(03)00553-6/pdf

6. Danetz, J, Cassano A, Stoner, M, Ivantur R, Levy M. Feasibility of endovascular repair in penetrating axillosubclavian injuries: A retrospective view, Journal of Vascular Surgery. 2005;41:246-54. Recuperado el 21 de abril de 2017, de http://www.jvascsurg.org/article/S07415214(04)01639-8/fulltext

7. Soto S, Sánchez G, Brousse J, Sánchez A. Trauma vascular periférico. Cuadernos de Cirugía. 2004;18:91-7. Recuperado el 20 de abril de 2017, de http://mingaonline. uach.cl/pdf/cuadcir/v18n1/art15.pdf

8. Castelli P, Caronno R, Piffaretti G, Tozzi M, Laganá D, Carrafiello G, et al. Endovascular repair of traumatic injuries of the subclavian and axillary arteries. International Journal of the Care of the 


\section{CASOS CLÍNICOS}

Injured. 2005:36:778-82. Recuperado el 10 de mayo de 2017, de http:// www.injuryjournal.com/article/S00201383(05)00004-5/fulltext

9. Fox C, Gillespie D, O'Donnel S, Rasmussen T, Goff J, Johnson C, et al. Contemporary management of wartime vascular trauma. Journal of Vascular Surgery. 2005;41:638-44. Recuperado el 22 de abril de 2017, de http://www.jvascsurg.org/article/S07415214(05)00032-7/fulltext

10. Ivantury R, Anand R, Ordonez C. Penetrating Extremity Trauma, World Journal of Surgery. 2015;39:1389-96. Recuperado el 18 de mayo de 2017, de http://sci-hub.cc/10.1007/s00268-0142865-8.
11. Reuben B, Whitten M, Sarfati M, Kraiss L. Increasing use of endovascular therapy in acute injuries: Analysis of the National Trauma Data Bank. Journal of Vascular Surgery. 2007;461222-6. Recuperado el 27 de abril de 2017, de http://www.jvascsurg.org/article/S07415214(07)01351-1/fulltext.

12. Branco B, DuBose J, Zhan L, Hughes J, Goshima K. Trends and outcomes of endovascular therapy in the management of civilian vascular injuries, Journal of Vascular Surgery. 2014;60:1297-307. Recuperado el 27 de abril de 2017, de http://www.jvascsurg.org/article/S07415214(14)01012-X/fulltext.

13. Fitridge R, Raptis S, Miller J, Faris I. Upper extremity arterial injuries:
Experience at the Royal Adelaide Hospital 1969-1991, Journal of Vascular Surgery. 1994;20:941-6. Recuperado el 20 de abril de 2017, de http://www.jvascsurg.org/ article/0741-5214(94)90231-3/fulltext

14. Desai S, DuBose J, Parham C. Outcomes after endovascular repair of arterial trauma, Journal of Vascular Surgery; 2014;60:1309-14. Recuperado el 22 de abril de 2017, de http://www.jvascsurg. org/article/S0741-5214(14)00997-5/ fulltext

15. Akyuz M, Gokalp O, Ozcem B. Surgical management of axillosubclavian vascular injuries, Pakistan Journal of Medical Science. 2015;31:552-5. Recuperado el 27 de abril de 2017, de https://www.ncbi.nlm. nih.gov/pmc/articles/PMC4485269/ 